\title{
Intoxications par le méprobamate : une préoccupation partagée par les réanimateurs, les toxicologues et les autorités sanitaires
}

\author{
Meprobamate poisoning: a common concern for critical care physicians, toxicologists \\ and the French health authorities
}

\author{
P. Saviuc $\cdot$ L. de Haro \\ (C) SRLF et Springer-Verlag France 2011
}

Le méprobamate est un psychotrope commercialisé en France depuis le début des années 1960, avec deux indications principales : anxiolytique en alternative aux benzodiazépines, notamment dans des contextes d'addiction (Équanil $^{\circledR} 250$ et $400 \mathrm{mg}$, Méprobamate Richard ${ }^{\circledR} 200 \mathrm{mg}$ ) et hypnotique (Mépronizine ${ }^{\circledR}$ associant deux principes actifs, $400 \mathrm{mg}$ de méprobamate et $10 \mathrm{mg}$ d'acéprométazine, une phénothiazine). Cette molécule, largement utilisée lors de conduites suicidaires, a été impliquée dans de nombreuses séries publiées de cas d'intoxication grave ou mortelle.

La première observation d'intoxication grave par méprobamate dans la littérature médicale date de 1956 [1]. Grâce à des publications nombreuses sur les surdosages en méprobamate, sa toxicité neurologique et surtout cardiovasculaire est bien connue : le choc vasoplégique ou cardiogénique est la principale complication mettant en jeu le pronostic vital. Des études épidémiologiques récentes ont montré que ce médicament est impliqué dans environ $7 \%$ des cas d'intoxication volontaire dans plusieurs pays, dont la France [2,3]. Depuis quelques années déjà, les centres antipoison et de toxicovigilance (CAPTV) français tirent la sonnette d'alarme et insistent sur le fait que le méprobamate demeure dans le peloton de tête des psychotropes pourvoyeurs de cas d'intoxication grave ou létale [4,5].

Ainsi, se basant sur les inquiétudes conjuguées de la pharmacovigilance, des toxicologues et des réanimateurs, l'Agence française de sécurité sanitaire des produits de santé (Afssaps) a requis plusieurs mesures visant notamment à réduire le risque d'intoxications graves. Dès 2006, les indi-

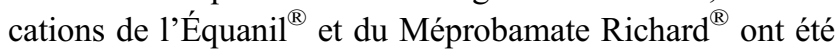

P. Saviuc $(\bowtie)$

Centre de toxicovigilance, CHU, BP 217,

F-38043 Grenoble cedex 09, Grenoble, France

e-mail : psaviuc@chu-grenoble.fr

L. de Haro

Centre antipoison et de toxicovigilance, hôpital Salvator,

Assistance publique de Marseille, France limitées au seul traitement de seconde intention de la dépendance alcoolique. Cette même année, l'Afssaps avait sollicité le comité de coordination de toxicovigilance (CCTV) pour proposer une actualisation de la rubrique 4.9 (surdosage) de la monographie du résumé des caractéristiques $\mathrm{du}$ produit $(\mathrm{RCP})$ présent dans le dictionnaire Vidal. À la réponse apportée en 2006 avait été ajoutée une note rappelant la préoccupation des CAPTV quant à la gravité des intoxications par le méprobamate, mentionnant particulièrement le grand nombre de cas graves d'intoxication par la Mépronizine $^{\circledR}$ rapportés aux CAPTV français.

Parallèlement, une diminution de la taille du conditionnement avait été proposée comme mesure de minimisation du risque dans le cadre d'une surveillance renforcée. Pour des raisons de modification des procédés de fabrication, l'application de cette mesure n'était prévue qu'à partir de 2009 pour l'Équanil ${ }^{\circledR}$, et l'Afssaps a annoncé en juillet 2009 la réduction de la taille du conditionnement de l'Équanil $^{\circledR} 250 \mathrm{mg}$ (de 7,5 à $5 \mathrm{~g}$ de méprobamate par boîte) et de l'Équanil ${ }^{\circledR} 400 \mathrm{mg}$ (de 12 à 4 g par boîte). Dans un second temps, un communiqué de presse de l'Afssaps a annoncé le 27 octobre 2010 la réduction de la taille du conditionnement de la Mépronizine ${ }^{\circledR}$ (de 12 à $2 \mathrm{~g}$ de méprobamate par boîte), cette mesure devant prendre effet début 2011.

\section{Où en sommes-nous en cette année 2011 ?}

Tout d'abord, comme la limitation des indications et la réduction du conditionnement sont effectives pour l'Équanil $^{\circledR}$ mais ne sont toujours pas appliquées pour la Mépronizine $^{\circledR}$, il était possible d'évaluer l'efficacité des décisions prises, en utilisant l'observatoire des CAPTV pour examiner l'évolution temporelle de la fréquence et de la gravité des intoxications par les deux spécialités. En réponse à une saisine de l'Afssaps, une étude rétrospective des données colligées par les CAPTV durant la période 2000-2010 
a été conduite. Les résultats de cette étude en cours de finalisation montrent une situation contrastée.

$\mathrm{Au}$ cours de cette dernière décennie, l'Équanil $^{\circledR 1}$ a été de moins en moins souvent impliqué dans les cas d'exposition notifiés, même après ajustement sur l'activité des CAPTV, quelles que soient les circonstances. Cette diminution a de fait accompagné la diminution des ventes. Elle était perceptible dès le début de la période d'étude, mais s'est accentuée depuis la limitation des indications survenue en 2006, l'interprétation de la diminution antérieure à 2006 étant plus difficile.

Le constat est un peu différent quand on s'intéresse aux seuls cas sévères : leur diminution parallèle à la diminution des ventes est plus marquée à partir de 2006 et pourrait en grande partie résulter des premières mesures de minimisation du risque. La réduction de la taille des conditionnements n'étant effective que depuis le milieu de l'année 2009, il est encore trop tôt pour en envisager l'évaluation; tout au plus peut-on noter l'amorce d'une diminution du nombre et de la proportion de cas sévères en 2009 et en 2010, ce qui devrait être confirmé ultérieurement.

La situation est différente pour la Mépronizine ${ }^{\circledR}$. Aucune mesure de gestion n'est effective à ce jour, et les ventes n'ont pas diminué. L'évolution du nombre de cas d'exposition est stable après ajustement sur l'activité des CAPTV. En revanche, si on s'intéresse aux cas sévères ou aux cas de conduite suicidaire, leur évolution montre une augmentation même après ajustement sur les volumes de vente. Ainsi, le nombre de cas sévères d'exposition à la Mépronizine ${ }^{\circledR}$ (tout comme le nombre de conduites suicidaires) croit régulièrement indépendamment de l'activité des CAPTV et de l'évolution des ventes.

Alors oui ! La limitation des indications de l'Équanil ${ }^{\circledR}$ voulue par les acteurs de la pharmacovilance et décidée par l'autorité de régulation a été efficace. Dans cette étude, pour les deux spécialités différentes, commercialisées pour deux indications différentes, qui ont subi des mesures de gestion différentes, les résultats indiquent des évolutions opposées en montrant que la Mépronizine ${ }^{\circledR}$ (pour laquelle les mesures préventives de réduction de conditionnement n'ont pas encore été appliquées) est bien plus impliquée dans les expositions sévères que l'Équanil ${ }^{\circledR}$ (pour laquelle des mesures de prévention ont été mises en œuvre). L'éventuel impact de la diminution de la taille du conditionnement devra être confirmé ultérieurement.

Alors non ! La perception des réanimateurs et des toxicologues quant à la fréquence importante des cas sévères,

\footnotetext{
${ }^{1}$ Le Méprobamate Richard ${ }^{\circledR}$, intervenant dans moins de $1 \%$ des ventes de méprobamate, a été dans cette étude regroupé avec l'Équanil ${ }^{\mathbb{R}}$. La diminution de conditionnement des boîtes est quant à elle intervenue durant le mois de décembre 2009 (de 10 à $4 \mathrm{~g}$ de méprobamate par boîte).
}

notamment après une exposition à la Mépronizine ${ }^{\circledR}$, n'était pas erronée.

Malgré les premiers résultats obtenus avec l'Équanil ${ }^{\circledR}$ grâce aux mesures de minimisation de risque, et qui restent à consolider, la question posée est celle du maintien sur le marché de spécialités anxiolytiques ou hypnotiques possédant une toxicité aiguë élevée avec un risque majeur d'intoxication grave et de décès lors de l'absorption de quantités importantes dans un contexte suicidaire. En effet, à partir des données collectées dans les CAPTV, l'analyse des expositions au méprobamate indique une proportion de cas sévères de $21,3 \%$, élevée en comparaison de celles établies dans les mêmes conditions pour l'ensemble des médicaments $(3,4 \%)$, le dextropropoxyphène $(7,6 \%$, retiré depuis 2011 [6]), le tramadol $(9,2 \%)$, le baclofène $(16,1 \%)$ [7], alors que des alternatives ne présentant pas de tels risques sont disponibles sur le marché.

Remerciements : L'étude d'évaluation des mesures de minimisation de risque à partir des données des centres antipoison français a été réalisée dans le cadre de la coordination de la toxicovigilance (animée par l'Institut de veille sanitaire, et regroupant notamment les CAPTV) et de son groupe de travail « médicament » (coanimé par l’Afssaps).

Les données des CAPTV proviennent du système commun des centres antipoison et de toxicovigilance d'Angers (P. Harry), de Bordeaux (P. Chanseau), de Lille (M. Mathieu), de Lyon (J. Descotes), de Marseille (B. Bruguerolles), de Nancy (J. Manel), de Paris (R. Garnier), de Rennes (C. Verger), de Toulouse (C. Cabot) et de Strasbourg (F. Flesch).

\section{Références}

1. Allen AG, Black AV (1956) Near-fatal case of intoxication with meprobamate treated with electro-stimulation and levarterenol. Ohio Med 52:1303

2. Charron C, Mekontso-Dessap A, Chergui K, et al (2005) Incidence, causes and prognosis of hypotension related to meprobamate poisoning. Intensive Care Med 31:1582-6

3. Blanc I, Tichadou L, Bourdon JH, et al (2007) Intoxications volontaires par le méprobamate. Suivi clinique et analytique des observations colligées au centre antipoison de Marseille au cours du $1^{\mathrm{er}}$ semestre 2006. Ann Toxicol Anal 19:151-2

4. Villa A, Cochet A, Guyodo G (2008) Les intoxications signalées aux centres antipoison français en 2006. Rev Prat 58:825-31

5. Fouilhé Sam-Laï N, Barral-Baron C, Groussin V, Saviuc P (2009) Recueil des intoxications graves et mortelles au CHU de Grenoble. Résultat des deux premières années d'étude, 2007-2008. $47^{\mathrm{e}}$ Congrès de la Société de toxicologie clinique, Toulouse 2-3 avril (http://www.toxicologie-clinique.org/stc2009/poster/fouilhe.pdf)

6. Saviuc P, Garnier R (2010) A-t-on raison de retirer du marché les spécialités à base de dextropropoxyphène ? Réanimation 19:466-70

7. Comité de coordination de toxicovigilance (CCTV) (2011). Méprobamate : impact des mesures de gestion sur le nombre de cas d'exposition notifiés dans les centres antipoison et de toxicovigilance. Rapport juin 